\title{
Functional Role of NAC Transcription Factors in Stress Responses and Genetic Diversity of Rice Plants Grown under Salt Stress Conditions
}

\author{
Mohamed A. El-Esawi \\ Botany Department, Faculty of Science, Tanta University, Tanta 31527, Egypt \\ * Correspondence: mohamed.elesawi@science.tanta.edu.eg
}

\begin{abstract}
Abiotic stress factors adversely affect crop growth and yield all over the world. NAC transcription factors could regulate stress response in plant species, and their underlying mechanisms should be studied. The current work investigates whether the rice transcription factor, $S N A C 3$, could augment salinity tolerance of rice plants. Results indicated that salt-stressed rice plants overexpressing SNAC3 had improved salt tolerance and yield, enhanced relative water content and osmolytes, increased gas-exchange attributes and antioxidant enzymes activity, up-regulated stressresponsive genes expression, and reduced oxidative stress markers levels, as compared to wild-type plants. SNAC3 rice mutants exhibited the reversed traits. Additionally, simple sequence repeats analysis showed genetically diverse patterns among treated and non-treated plants. In conclusion, SNAC3 has a crucial role in improving salinity tolerance and grain yield of rice plants. This study also presented transgenic rice lines with enhanced grain yield and improved salinity tolerance.
\end{abstract}

Keywords: NAC transcription factor; salinity; traits; antioxidants; genes expression. 